\title{
Inmigrantes de Bolivia en el colegio en São Paulo: fronteras del derecho a la educación*
}

\author{
Giovanna Modé Magalhães**
}

Flávia Schilling***

* Originalmente publicado en la Revista Pro-Posições, 23(67),43-64, jan./abr. 2012

* Maestra en Sociología de Educación de la Facultad de Educación de la Universidad de São Paulo, Brasil. giovannamode@hotmail.com

*** Profesora de la Facultad de Educación de la Universidad de São Paulo, Brasil. oakl@uol.com.br 
Traducciones

\section{Inmigrantes de Bolivia en el colegio en São Paulo: fronteras del derecho a la educación}

\section{Resumen}

Entender cómo está siendo implantado el derecho humano a la educación para los y las inmigrantes de Bolivia que viven en São Paulo es el principal objetivo de este estudio, que se inscribe en dos debates globales contemporáneos: de un lado, los que abarcan la complejidad de las migraciones internacionales y, de otro lado, los que tratan las tensiones relativas a la universalización de derechos en sociedades desiguales y discriminatorias. A lo largo de la investigación, analizamos la legislación nacional e internacional y recorremos la literatura contemporánea sobre migraciones internacionales y sus puntos de contacto con el campo de los Derechos Humanos, especialmente, al problematizar la relación entre los derechos de todos y los derechos de los ciudadanos puesto que muchos de los que hacen parte del grupo estudiado viven en situación irregular en Brasil, lo que no les quita la garantía de la realización de los derechos fundamentales-. Para una mejor comprensión de esta dinámica, visitamos lugares de convivencia de los inmigrantes bolivians(as) en la ciudad, tuvimos encuentros con organizaciones que trabajan el tema y finalmente, hicimos 16 entrevistas con estudiantes, madres, padres, profesores(as) y funcionarios de escuelas públicas de la capital. 


\section{Introducción}

Nuestra investigación ${ }^{1}$ tuvo como objetivo principal, discutir cómo está siendo implementado el derecho humano a la educación escolar para los (las) inmigrantes de Bolivia que viven en São Paulo. Para esto, dos debates contemporáneos estuvieron presentes: por un lado la creciente complejidad de las migraciones internacionales y, por el otro, las tensiones relativas a la universalización de derechos en sociedades desiguales y discriminatorias. Buscamos observar cómo es el acceso a la educación escolar: ¿cómo pensar la educación de personas desenraizadas, como recuerda Martins (2001), en una de las instituciones más territorializadas de la modernidad?

São Paulo está entre las grandes ciudades receptoras de inmigrantes, su dinámica continúa atrayendo no solo brasileros(as) de diferentes estados, sino también extranjeros(as), de una forma diferente que las grandes migraciones que caracterizaron el siglo XIX y la primera mitad del siglo XX. Con nuevos entornos e inmersa en un contexto global bastante diferente, la metrópoli tiene uno de los flujos de inmigrantes más importante, originado en los países vecinos, entre ellos, el mayor es el de bolivianos(as) ${ }^{2}$.

Se trata de la migración de personas que encuentran trabajo en talleres textiles en la ciudad, estimadas entre 10 mil y 12 mil, concentradas principalmente en la región central $^{3}$. Son hombres, mujeres, jóvenes, niños, ancianos, trabajando en condiciones precarias. El reporte de una $\mathrm{CPI}^{4}$, en 2005, mostró que entre los problemas enfrentados están: dificultades de acceso a los servicios públicos, condiciones de trabajo insalubres e intolerancia de la población local con relación a los hábitos, costumbres e idiomas diferentes.

1 Fronteiras do Direito Humano à educação: um estudo sobre os imigrantes bolivianos nas escolas públicas de São Paulo. São Paulo: Faculdade de Educação da Universidade de São Paulo, 2010.

2 El número de población boliviana que vive en Brasil es una incógnita. Los datos del Censo 2000 contabilizaban 20.015 bolivianos viviendo en territorio brasilero. Ya la Policía Federal hablaba de 32.416 personas legalizadas. El consulado de Bolivia habla de 50 mil indocumentados, mientras que para la Pastoral del Inmigrante el número está entre 70 mil y 80 mil. El Ministerio de Trabajo y Empleo tiene cálculos menores —entre 10 mil y 30 mil—. El Ministerio Público habla de 200 mil bolivianos en total (incluyendo regulares e irregulares).

3 Los relatos de las organizaciones de la sociedad civil que trabajan sobre este tema, coinciden en mencionar que viven en los barrios donde está ubicada la industria de costura, la mayoría de la región central. Sin embargo, la investigación realizada recientemente por Cymbalista y Rolnik (2007), apunta a un fenómeno de desconcierto en dichos talleres, que podría deberse a razones de seguridad o de búsqueda de arriendos más baratos.

4 Los relatos de las organizaciones de la sociedad civil que trabajan sobre este tema, coinciden en mencionar que viven en los barrios donde está ubicada la industria de costura, la mayoría de la región central. Sin embargo, la investigación realizada recientemente por Cymbalista y Rolnik (2007), apunta a un fenómeno de desconcierto en dichos talleres, que podría deberse a razones de seguridad o de búsqueda de arriendos más baratos. 
En la red municipal de educación paulista, existen hoy 1.446 alumnos y alumnas nacidos en Bolivia (datos del 2009), aparte de los que están matriculados en la red estadual ${ }^{5}$ y de los que están en edad escolar y fuera de cualquier escuela. Indagaremos cómo los(as) inmigrantes bolivianos(as) están involucrados en los sistemas educativos, cómo construyen una relación con la comunidad escolar local, cuáles son las características que han marcado esa trayectoria escolar y cuál es el sentido de ese paso por la escuela brasilera para ellos y ellas. ¿Ese derecho universalmente consagrado para todas las personas ha sido llevado a cabo en este caso? Internacionalmente observamos un crecimiento de los estudios sobre las dinámicas migratorias, principalmente en los países receptores de inmigrantes, y uno de los múltiples enfoques trabajados es la presencia de los más diversos grupos extranjeros en los sistemas educativos locales. Esas investigaciones exploran cuestiones pedagógicas, como la preparación de los profesores para lidiar con la diversidad, los desafíos de los idiomas, la dificultad del aprendizaje, entre otros. Existen incluso, investigaciones que tratan de las relaciones con la familia, de las diferencias culturales, de la discriminación y del prejuicio en la escuela. Reafirmamos la importancia del tema como un problema social, orientando una serie de investigaciones.

En Brasil, en una pesquisa sobre el estado del arte de investigaciones sobre las migraciones internacionales y el derecho a la educación, encontramos pocos estudios académicos. Tal vez, esta realidad retrata una situación inversa a la observada en otros lugares del mundo, la cuestión parece permanecer cercada por una invisibilidad social y vista como algo sin importancia, todavía sin despertar gran interés. Sin embargo, nos encontramos, con un creciente interés por el estudio de la población boliviana en São Paulo, en especial por lo que se dice respecto a la dimensión del trabajo en los talleres de costura ${ }^{6}$.

Además de la investigación bibliográfica, a partir de la indagación del tema en los medios impresos, del análisis de los resultados de la mencionada CPI y del análisis de la normativa nacional e internacional, realizamos visitas a los principales lugares de sociabilidad de los bolivianos(as) en São Paulo. Visitamos espacios de convivencia, como la Praça Kantuta, aparte de las canchas de fútbol y la radio comunitaria, así como recorrimos instituciones, la mayoría de carácter gubernamental, que conforman una red de apoyo a los inmigrantes bolivianos en São Paulo? .

5 Este número no está disponible.

6 Destacamos, entre otras, la valiosa contribución de Silva (1997, 2003, 2006 y 2007).

7 Entre ellas, se destacan la Pastoral do Imigrante, la Casa do Migrante, el Centro de Estudos Migratórios (CEM), el Centro de Apoio ao Migrante (CAMI) y el Serviço Pastoral do Migrante (SPM). Silva (2005) las principales asociaciones y grupos de manifestaciones culturales en São Paulo. Cita la Associação dos Residentes Bolivianos (ADRB), responsable de la publicación mensual La puerta del Sol. El Círculo Boliviano fue fundado en 1975; en 2001 surgió la Associação comercial Brasil Bolívia (Bolbra), entre otras. 
Finalmente, hicimos entrevistas con un director, una asistente de dirección, una profesora y dos profesores en una escuela municipal ubicada en el barrio de Pari, y con madres de estudiantes. En total, participaron en las entrevistas grabadas, de manera individual o colectiva, 16 personas. En una entrevista-piloto, realizada con una profesora de un colegio municipal, fueron relatados problemas de las más variadas naturalezas (idioma, burocracia, documentación), dificultades de diálogo, indicios de discriminación y un silencio alarmante.

Diseñamos tres ejes analíticos, que guiaron el trabajo de análisis y la presentación de entrevistas. El primero de ellos, fue el acceso y la permanencia en el colegio. Miramos, principalmente, la cuestión de la documentación exigida, así como la relación con el idioma nativo. ¿La educación es completamente disponible y accesible para la población inmigrante boliviana? En el segundo eje de análisis, la propuesta fue observar la relación entre los y las inmigrantes bolivianos(as) y sus familias con la comunidad escolar local y los detalles del proceso de diálogo e integración. ¿Cómo, por ejemplo, los hijos(as) de inmigrantes nacidos en Brasil se veían frente a la doble nacionalidad formal? ¿Brasileros(as) o bolivianos(as)? Aquí aparecen las cuestiones de la diferencia, la discriminación y la violencia. ¿La educación es aceptable y adaptable para esos extranjeros? Finalmente, el tercer eje de análisis fue diseñado para conocer las familias de inmigrantes bolivianos(as), incluyendo los mismos alumnos(as), pero no solo ellos, las madres se mostraron como interlocutoras privilegiadas. ¿Cuáles eran sus expectativas?, ¿cómo veían la educación brasilera?, y ¿cuál era el sentido de esa educación para los inmigrantes?

\section{Inmigrantes: contornos del debate}

Para el análisis de la situación de los inmigrantes de origen boliviano en los colegios de São Paulo, nos pareció pertinente enfocarnos en tres debates históricos. El primero es el que gira en torno a las dinámicas migratorias contemporáneas. La Organización Internacional para las Migraciones de las Naciones Unidas ${ }^{8}$ mostró que más de 200 millones de personas viven fuera de sus países de origen. Sayad (1998) dice que se trata de un "hecho social completo", un fenómeno colectivo e individual que debe ser observado desde el cruce de las ciencias sociales con las contribuciones de distintas disciplinas, como historia, geografía, demografía, economía, derecho, sicología, antropología y política. lanni (2004), para referirse a las migraciones contemporáneas, trabaja con el concepto de "transmigración", que engloba la heterogeneidad que caracteriza a la experiencia migratoria actualmente. A los que migran por primera vez, se suman los descendientes de inmigrantes, intensificando tensiones, crisis y conflictos, y al mismo tiempo, significados, vivencias y horizontes, que van imponiendo, ampliando y multiplicando las experiencias de transculturación, pluralidad y relatividad en los países occidentales (Ianni, 2004, p. 93).

8 Internationa I Organi zation for Migration (IOM). World Migration 2008: Managing Labour Mobility in the Evolving Global Economy. Ginebra. 
Así, una segunda vertiente conceptual que se hace necesaria en nuestro estudio, son los debates recientes sobre las diferencias culturales y sus conexiones con una sociedad desigual, dentro y fuera de los sistemas educativos, ya que, en este caso, las paredes del colegio son una frontera porosa. El inmigrante trae consigo sus referencias, su religión, su idioma, sus costumbres y, en algunos casos como el de la población boliviana en São Paulo, trae también características étnicas. Él es, en todo caso, un sujeto diferente. Así, existe un reavivamiento de las diferencias, circunscribiendo el análisis de las tensiones contemporáneas, como las investigadas por Touraine (1999), cuando pregunta si, podremos vivir juntos. Con el debate sobre las diferencias, Boaventura de Sousa Santos (2006) suma el factor de la desigualdad, que nos parece que es una dimensión fundamental para entender el lugar de las discriminaciones vividas por las poblaciones migrantes, especialmente si se tiene en cuenta que nuestro grupo en análisis ocupa la parte inferior de la escala socioeconómica9 .

Finalmente, la tercera vertiente analítica en la cual se inscribe esta investigación está en las disputas y tensiones entorno al campo de los Derechos Humanos. Entre las varias posibilidades, dos ejes orientaron nuestro estudio: la relación entre ciudadanía, Derechos Humanos e inmigración, con especial énfasis en las contradicciones entre los derechos de todos y los derechos de los ciudadanos, y por otro lado, la discusión sobre cómo se define el derecho humano a la educación. Podemos afirmar, que existe un consenso sobre la necesidad de garantizar a esos no ciudadanos el amparo necesario hasta que su situación sea regularizada (Lafer, 1988). A pesar de esto, ese ha sido un punto de tensión clásico no solamente en el análisis, sino también en las políticas contemporáneas. ¿Cómo lidiar con una población que un determinado estado no quiere recibir, y que en últimas, ya está en su territorio? La segunda discusión en el campo de los Derechos Humanos es justamente la que define cómo se da la implementación del derecho a la educación. Aunque hay un gran acuerdo internacional sobre la necesidad de una "educación para todos", la comprensión de quién está en ese "todos" y de cómo se realiza el derecho a la educación, también es un campo de disputa.

En su Observación General n ${ }^{\circ} 13$ sobre el Derecho a la Educación, el Comité de Derechos Económicos, Sociales y Culturales (DESC) de las Naciones Unidas afirmó, en 1999, algunos criterios para la realización de los derechos educativos, ${ }^{10}$ que se llevan a cabo cuando la educación está disponible y es accesible, aceptable

9 Como ilustró una de nuestras entrevistadas, M2, que vino de Potosí y estaba en São Paulo hace siete años: Llegamos sin nada. Mi marido trabajaba en una empresa, pero lo echaron. Entonces no teníamos dinero, y mi marido no tenía trabajo en Bolivia y usted sabe que a la mujer no la dejan trabajar. Mi hijo más pequeño tenía 8 años. Cuando yo llegué aquí, buscábamos trabajo y no sabíamos coser. Pero fuimos a trabajar en un taller de costura de un boliviano, un compatriota mío, que nos esclavizaba, no nos pagaba. Ahora ya estoy mejorando, niña, gracias a Dios y gracias a São Paulo.

10 En el período 21 de sesiones y acogiendo el consejo de la entonces relatora especial de la ONU para el derecho a la educación, Katarina Tomasevski. 
y adaptable. Disponible en el sentido de que hayan instituciones y programas de educación en suficientes cantidades; accesible, sin obstáculos económicos, legales o discriminatorios; aceptable, que cuente con patrones mínimos de calidad; y adaptable en relación a la flexibilidad necesaria para responder a las necesidades de los estudiantes, en los más diferentes contextos sociales y culturales. Esta propuesta fue reconocida internacionalmente como el modelo de las "4 As" (por sus siglas en inglés: available, accesible, acceptable, adaptable) y viene siendo utilizada como forma de verificar la realización de este derecho.

Los inmigrantes han encontrado obstáculos relacionados con la accesibilidad (la documentación exigida es una barrera evidente), la aceptabilidad (la discriminación), la adaptabilidad (diferencias ignoradas, comenzando por la lingüística. Los relatos mostrarán la opinión sobre la aceptabilidad,calidad). ¿La educación de los(as) bolivianos(as) está, en realidad, disponible y es accesible, aceptable y adaptable?

Los derechos educativos volvieron a ser reafirmados en las normativas internacionales que tratan específicamente sobre las poblaciones de migrantes y refugiados. La Convención internacional sobre la protección de los derechos de todos los trabajadores migratorios y de sus familiares ${ }^{11}$ dice, en su artículo 30, que el hijo de un trabajador migrante tiene el derecho fundamental de acceso a la educación, en condiciones de igualdad de trato respecto a los nacionales del Estado receptor. En Brasil, la Constitución Federal de 1988 es, ciertamente, un marco de referencia para la educación escolar, al declarar toda la educación básica como derecho subjetivo y exigible por cualquier persona en los sistemas de justicia. En 1990, fue aprobado el Estatuto del niño y del adolescente (ECA) ${ }^{12}$ que, en su artículo 53, garantiza "igualdad de condiciones para el acceso y permanencia en la escuela".

Sin embargo, el anacronismo del Estatuto del Extranjero (Ley 6815/80) ha venido causando problemas de distintas naturalezas para la realización de los derechos de esas poblaciones. Dicha ley dice que los colegios solo pueden hacer efectiva la matrícula, en cualquier grado de educación, de los extranjeros "debidamente registrados" (art. 48). Con base en esta premisa, en enero de 1990, fue aprobada la Resolución n. 9 por la Secretaría de Educación del Estado de São Paulo, que prohibía los niños sin documentos frecuentar la escuela, particular o pública. El documento decretaba que los establecimientos de educación debían exigir el Registro Nacional de Extranjero, RNE, proporcionado por la Policía Federal, y enviar la documentación a las Coordinaciones de Educación ${ }^{13}$, que tenían la obligación de entregar la relación de los estudiantes a la Secretaría de Educación, órgano que, a su vez, debería enviar un reporte con la información al Ministerio de Justicia.

11 Brasil todavía no ratificó esta convención.

12 Ley Federal N. 8.069 / 90.

13 Por medio de las Delegaciones de Educación y de las Divisiones Regionales de Educación. 
Antes de esta ley, los(as) alumnos(as) extranjeros(as), incluso aquellos(as) en situación irregular, venían frecuentando normalmente el colegio (Bonassi, 2000, p. 173). Se estima que cerca de 400 niños y adolescentes hayan dejado el colegio en dichas circunstancias en el estado de São Paulo. Frente a este escenario, algunas organizaciones de la sociedad civil, entre ellas la Comisión de Justicia y Paz y el Centro Pastoral de los Migrantes, pidieron formalmente la revocación de la Resolución N. 9. En conjunto, estas organizaciones elaboraron un dossier ${ }^{14}$ presentado, en febrero de 1994, al Ministerio Público. En el año siguiente, a comienzos de 1995, fue anulada la resolución $\mathrm{N}^{0} 9$ y anunciada la $\mathrm{N}^{0} 10^{15}$, reforzando justamente el derecho a la educación para los niños y los adolescentes extranjeros, independientemente del estatus legal en el que se encontraban en el país. Hasta hoy, han sido registradas dificultades en este sentido, a pesar de que la cuestión del acceso al colegio se haya visto como resuelta.

En el ámbito estadual, dos dictámenes reafirmaron la Resolución, uno en 1997 y el otro en el $2008^{16}$. El segundo, además de resolver la situación en cuestión, explicitó que: "se deberá ofrecer al alumno extranjero todo el apoyo pedagógico necesario para su adaptación, para que pueda acompañar los contenidos curriculares de la base nacional común, especialmente a lo que se refiere al aprendizaje de la Lengua Portuguesa"17. En 2004, la población inmigrante que residía en la ciudad de São Paulo, comenzó a contar con otro dictamen, esta vez del Consejo Municipal de Educación ${ }^{18}$.

\section{Bolivianos(as) en los colegios}

Frente a estas cuestiones teóricas del panorama normativo encontrado, emprendimos el trabajo de campo. Como mencionamos anteriormente, el resultado de las entrevistas fue organizado en tres ejes analíticos: un primer eje que trató del acceso y de la permanencia en el colegio; el segundo, que abordó las relaciones establecidas en el ambiente escolar; y el tercero que trató sobre las expectativas de las familias bolivianas con relación a la trayectoria escolar.

\section{Documentos, idioma: las primeras fronteras del derecho}

En el primer eje, nos preguntamos de qué manera se configura el derecho a la educación. ¿La educación está disponible y es accesible para todos y todas? Una primera cuestión es verificar las posibles barreras en el acceso a la escuela, en lo 14 Dossiê-Escola, Centro Pastoral dos Migrantes, 1994.

15 El abogado Belisário dos Santos Júnior, el entonces Secretario de Justicia y Defensa de la Ciudadanía, por medio de la Comisión de Justicia y Paz, pidió la anulación de la Resolución al gobernador (Bonassi, 2000, p.179).

16 Conselho Estadual de Educação, Deliberação 16, 1997; Conselho Estadual de Educação, n 633/2008.

17 En Bolivia, 74\% de la población es considerada indígena, la mayoría de origen quechua (30\%) y aimara (25\%). En el país son hablados 26 idiomas y 127 dialectos (Silva, 2005).

18 Conselho Municipal de Educação, Parecer 17, 2004. 
que concierne a aquellos alumnos(as) inmigrantes en situación irregular en Brasil. La forma más explícita de violación sería exigir una documentación que esa población no tiene y condicionar a esta documentación tanto la matrícula, como la emisión del certificado de conclusión del curso.

Aunque las dificultades relacionadas con el ingreso estén lejos de aquellas narradas en los inicios de los años de 1990 (Bonassi, 2000, p. 175), notamos que la matrícula no es un obstáculo totalmente superado. Así se confirme que la presencia de bolivianos(as) aumentó en los colegios y que la información sobre el derecho a la matrícula está más expandida, el problema no está resuelto. Explícito en la legislación, en los niveles internacional, nacional y local, y reafirmado en las resoluciones de los consejos estadual y municipal de educación, el problema gana un aspecto de falta o equivocación de informaciones. En nuestro estudio, contactamos las secretarías de las unidades escolares que exigen un documento que esta población no tiene. Encontramos estudiantes potenciales, y familias que no encuentran información sobre cómo continuar sus estudios. La primera impresión, en este caso, fue que las madres o los estudiantes bolivianos(as) no suelen hacer un segundo intento o insistir en hacer efectiva la matrícula, hecho bastante normal, dada la vulnerabilidad de las personas que están sin documentación regular y que, finalmente, pueden sufrir sanciones o ser deportadas. En vez de esto, la tendencia es continuar en primer lugar la batalla por la regularización en el País, como ilustra el comentario de la madre M2:

Mis hijos tienen el problema que todavía no pueden estudiar, porque ellos todavía no tienen el documento. Nosotros queremos renovar el documento, la permanencia, pero ellos nos dicen que tenemos que esperar. [...] Ahora mi otro hijo también quería hacer el estudio de conductor, y tampoco puede, ¿por qué? Porque no tiene documentos ${ }^{19}$.

En la entrevista de E3, joven de 20 años que intentó varias veces matricularse en cursos técnicos de la red pública e incluso en cursos libres de computación de la iniciativa privada, nuevamente quedan explícitas dichas barreras:

Es mucha burocracia [...] El documento provisional no vale, quieren el de permanencia. Ahora lo van a renovar otra vez dentro de 180 días. Siempre lo renuevan, pero siempre quedamos con otro provisional. [...]. Los secretarios de las escuelas no saben cómo informar y no me dejan hacer los cursos.

El no conocimiento de los derechos se hizo notar también en una perspectiva más amplia. La madre M2 mencionó que "pidió el favor" para que el hijo consiguiera la matrícula:

19 En el original las transcripciones del habla de las personas entrevistadas como fueron grabadas, exactamente para mostrar la cuestión crucial del idioma. Sin embargo, en esta traducción no es posible conservar estas características del habla de los migrantes bolivianos, debido a que se hace entre los dos idiomas que ellos combinan para expresarse (N. del T.). 
Mi segundo hijo pudo estudiar en un instituto de computación, les pedimos el favor. [...]. El instituto era de un amigo mío boliviano y, como somos de Bolivia, él lo dejó. [...]. Pero en los institutos aquí de Brasil, la gente no quiere saber nada, no quiere. Y también el año pasado hablé con la directora aquí del colegio, para que mi hijo terminara de estudiar [...]. Y entonces, la directora me dijo: ¿dónde están los documentos? Y yo le dije a la directora: Hazme el favor, él quiere estudiar, yo le dije, hazme el favor. Yo le lloré, niña, lloré, entonces ella aceptó, gracias a Dios aceptó, niña.

Esta barrera apareció explícitamente en la declaración del director que entrevistamos, que confirmó la dificultad al decir que: "La madre viene a veces al colegio y en la secretaría exigen la certificación de nacimiento, pero nosotros sabemos que por la legislación de hoy no es necesario". Sin embargo, él no levantó ninguna medida para solucionar esta cuestión. Entre madres, estudiantes, o personas en búsqueda de oportunidades educativas, nadie conocía la legislación y los derechos educativos de la población migrante. No hubo referencia a ningún lugar donde pudieran encontrar este tipo de orientación.

Otro aspecto fue el de la relación español-portugués. De todas las personas con las que conversamos, ninguna ingresó inmediatamente al colegio en el momento en que llegó a São Paulo, incluso las personas que relatan una historia exitosa, cuentan que hicieron una pausa, en la mayoría de los casos con un año de duración, motivada por la inseguridad con el nuevo idioma. El lenguaje aparece como una barrera inicial para todos(as) los que inmigraron. En la ausencia de espacios de sociabilidad y convivencia con brasileros(as), la principal aliada para el aprendizaje del idioma, cuentan, es la televisión: "Yo dejé de estudiar un año por la escritura, pero después entré en la quinta serie [...]. Al comienzo, aprendí con canciones, mi mamá compraba revistas y la televisión ayudó mucho. Por eso no fue fácil" (E5, 17 años). E4, joven que entró en un grupo de EJA a los 19 años, confirma: "Todo está bien, solo tengo el problema de la escritura [...] solo tengo esa dificultad con el portugués [...]. Nosotros miramos mucha televisión. También leemos. Pero a veces empezamos a decir algunas cosas, que en el momento que vamos a ver, están mal".

Excepto iniciativas puntuales de clases de portugués para inmigrantes adultos, no encontramos programas que apuntaran específicamente a un esfuerzo para los alumnos(as) que tienen el español como idioma nativo, a pesar de que la constatación de la dificultad con la lengua escrita, aparezca claramente en las entrevistas con los profesores:

Ellos dominan la lengua oral, porque la escrita es una dificultad. Hay cosas que vienen del español. Entonces ellos escriben una frase interrogativa y ponen el signo de interrogación al revés. Yo les digo, mira, hasta tiene sentido, pero no existe en portugués. Cambian las letras, la B y la $\mathrm{V}(\mathrm{P} 1)$. 
En el caso de aquellos estudiantes o familias que explícitamente quieren volver a Bolivia, hay otro sentido: el objetivo sería mantener el español, como recuerda M3, madre de dos niños y dos niñas. Ahí nace la decisión de mandar sus hijos a estudiar en su país de origen: "Yo que no estoy de estar para siempre aquí, tengo que salir de aquí en unos cuantos años, y es por esto que los mandé allá, es un poco complicado el idioma, perjudica, mejor que estudien allá".

Es una realidad que, en el ámbito privado, la gran mayoría parece mantener el idioma. Asimismo encontramos un caso de una familia, que reside en São Paulo hace siete años, en la que la madre le pedía a la hija E5, de 17 años, que solo hablara portugués en casa, pues trabajando en el taller de costura y permaneciendo horas en casa, ella tendría poco tiempo para aprender el idioma. Situación semejante a la relatada en el pasaje Folclore e mudança social na cidade de São Paulo, en el que Floresta Fernandes (1961) menciona la contribución de los niños inmigrantes de São Paulo en el proceso de socialización de los padres, a mediados del siglo XX.

\section{Invisibles, silenciosos: segunda frontera del derecho}

El segundo eje de análisis trata sobre la relación entre bolivianos(as) y sus familias con la comunidad escolar local, personal directivo, profesores y otros alumnos(as) brasileros(as). Destacaremos los relatos que evidencian las tensiones de lo cotidiano, y nos enfocaremos en la percepción de la diferencia, la discriminación y la violencia. Desde el comienzo de la investigación, los(as) bolivianos(as) eran descritos como estudiantes silenciosos(as) y, muchas veces, invisibles dentro de las escuelas. Ese silencio y esa invisibilidad parecían permitir muchos sentidos y ambivalencias que aparecieron en las entrevistas. Son, así, descritos como alumnos que hablan pasito, silenciosos, tímidos. Esta descripción era común en las versiones de los profesores. El director D1, por ejemplo, no sabía el idioma que los alumnos hablaban entre ellos: "Nunca entendí, lo que sé es que hablan (español) con la mamá; la madre a veces tiene más problemas con el portugués". El profesor P2 trae como primera evidencia la diferencia que nota en el comportamiento de los niños: "Para nosotros eran diferentes, por causa del comportamiento que es diferente al de los brasileros. Los brasileros son muy efusivos y los bolivianos más retraídos, bastante más retraídos". Dentro del salón de clase esta características se expresaba de maneras diversas, como relató la profesora $\mathrm{P} 1$ :

Yo sé que ellos tienen dominio del portugués, pero tienen miedo de exponerse. Entonces hablan muy poco, hablan muy bajo, hay unos que ni responden cuando los Ilaman. [...]. Y no preguntan. Ellos no hablan, entonces hay alumnos a los que les conozco la voz, porque yo voy y hablo con él, si no, no lo escucharía nunca. Usted pregunta: “¿Usted vive aquí en la esquina?", él responde: "No sé". Ellos dicen "no sé" para la mayor parte de las preguntas que usted hace. (...) Después yo hice algunos seminarios, pero él no participaba. Él prefería sacar "cero" que hablar. 
En las entrevistas con las madres y los(as) estudiantes bolivianos(as), el silencio y la timidez aparecieron de dos maneras, por un lado, como una cualidad, una forma de respeto al ambiente escolar que trae consigo una reverencia a la figura del profesor. Por otro lado, la misma característica fue relatada por otros(as) como un problema que hay que superar, sería una timidez responsable por la falta de defensa frente a las agresiones verbales cotidianas que más tarde vivieron. El primer significado, por ejemplo, aparece en el habla de E5, joven boliviana, de 17 años, cuando dice que:

Los profesores nos elogian mucho a nosotros, los bolivianos, somos bien educados en el salón, no peleamos con el profesor, es porque estudiamos, sacamos notas. Entonces ellos (los brasileros) cuando escuchan eso, dicen: "ah, usted es muy juiciosito, tiene que hacer desorden".

Algunos profesores reconocen que "la mayoría está formada por alumnos promedio o muy buenos" y se arriesgaron a dar una interpretación, aunque con dudas y desconfianza, como P3: "Creo que ese silencio no es timidez, sino una forma de reverencia". Al mismo discurso de E5 que comentamos arriba, un estudiante de tercer año de Educación Media aportó una segunda manera de ver esa misma característica, cuando relató una situación en la que tuvo que responder a la provocación de compañeros que molestaban a la joven boliviana y a otros amigos bolivianos: "Porque ellos son tímidos... hay algunos que te molestan, pero si no les respondes ahí mismo, ellos van a seguir molestando, me parece". La misma percepción salió enfáticamente en la entrevista de M3, madre, cuando ella insistió:

El boliviano siempre es tímido, tiene miedo de hablar, no habla nada, se calla [...]. Es de jóvenes que yo les decía [a los hijos] que tienen que saber defenderse, que tienen que saber hablar. Yo siempre les enseño que todos somos iguales. Digo que no hay miedo. Solo que las madres bolivianas no hacen eso. No hablan mucho, hacen que ellos tengan miedo, se quedan en la propia casa encerrados, donde trabajan papá y mamá.

Este aspecto, ese notable silencio nos conduce a otra reflexión, muy presente en la conversación con los profesores, el director y otras personas no inmigrantes dentro y fuera del colegio: dice mucho en relación al poco conocimiento que se tiene sobre la población de origen boliviana que vive en São Paulo. "No conozco, no sé bien, creo que, parece, no estoy seguro" fueron expresiones que aparecieron recurrentemente. Un ejemplo es la entrevista de la profesora P1:

En todas las clases, tenemos al menos uno. Y en general no solo uno, son dos, tres... Llegan al $10 \%$ de la clase. Apenas llegué me impactó eso, dije: "¿parece que hay una comunidad de esas aquí? [...]". Pensé que [los demás profesores] conocerían algo sobre ellos, pero a lo largo del tiempo vi que no, ellos me contaron que había una feria, pero la verdad la mayor parte de los profesores ni fue nunca a esa feria. 
La profesora P1 evidencia una notable incomodidad cada vez que intenta hablar sobre el tema de la inmigración en el salón de clase:

Quedan muy incómodos. [...] Yo no sé por qué [...] yo mismo conozco muy poco de Bolivia, del tema de los bolivianos en Brasil también muy poco. [...] Hasta intento, pero ahí veo que ellos quedan tan incómodos. Y entonces un día solo llamé a algunos "¿Ustedes quieren que yo no hable de eso?" y dijeron "No quiero, quiero que no hable de eso, hable de otras cosas."

Otro pasaje interesante del relato de esta misma profesora es sobre una experiencia con el grupo de octavo grado, en el que intentó hablar de la xenofobia y de las migraciones de otros países, ya que muchos alumnos(as), brasileros(as), en este caso, expresaron de alguna manera su deseo de migrar:

Ellos tienen la idea, tienen el sueño de ir a Estados Unidos, y de que como inmigrante van a llegar allá con un estatus de reconocimiento, van a ganarse la vida. [...] Entonces yo pregunto: “¿Usted trata bien al extranjero?" "No, pero conmigo va a ser diferente, porque yo me voy a mezclar". "¡No va a ser así! Usted cree que tiene la cara". Y nos quedamos en esa discusión. Y en la última clase en la que tuvimos esa discusión, hay una boliviana en la clase, y ella reía, reía... Ella nunca se había manifestado, ella normalmente se queda quieta.

Otra declaración que ilustra estas ambivalencias es la de E5, de 17 años. Cuenta que, cuando llegó, hace siete años, no tuvo dificultades para integrarse, era la única boliviana en el salón de clase en aquella época y fue bien recibida por los colegas. Sin embargo, comenta un posible cambio en esta dinámica en los días de hoy:

Estuvo muy bien, recibí el apoyo de todos, porque era la única boliviana en el salón, y un montón de brasileros. Ellos me miraban así y decían "Dios mío, ¿usted habla español?" y venían a preguntarme un montón de cosas de mi cultura. Preguntaban cómo era allá, cómo era Bolivia. Y yo decía que era un lugar bonito, pero que era frío y ellos decían "ah, yo quiero ir allá [...]. Los profesores también fueron buenos. Pero, sabe, de ese tiempo para acá cambiaron muchas cosas. Ahora hay, sí, un prejuicio contra los bolivianos que llegan a Brasil.

Frente al desconocimiento, por un lado, y la discriminación por otro, emergían indicios de un encuentro, de cierta manera en conflicto, en uno de los únicos lugares donde la convivencia entre inmigrantes y nativos se daba, el colegio.

Entre nuestras preguntas iniciales, existía una duda sobre cómo los hijos de los inmigrantes bolivianos, en el caso de los nacidos en Brasil, se consideraban en relación a la doble nacionalidad. Habíamos notado, en la conversación con los profesores que pasar a ser identificado como brasilero era una forma de resolver parte de los conflictos, ellos entrarían en una zona más segura, como contó P3: "Hay algunos a los que usted les pregunta: “¿usted es boliviano?", y él dice "no, yo soy 
brasilero, mi papá es el que es boliviano", como si fuera un problema. Como decían los nordestinos antes". Daban mucha importancia al uso del portugués, en especial en los lugares públicos. En el caso de los descendientes nacidos en Brasil, todos se definían como brasileros. M4, madre de una niña que nació en Brasil y hoy tiene 12 años, afirma:

Ella dice que no es boliviana, siempre dice que nació aquí. A ella no le gusta que yo digo que ella es boliviana, no sé por qué, pues desde pequeña ella estuvo con los bolivianos. Una vez le dije que ella era boliviana y ella dijo que no, que la que nace en Brasil es brasilera.

La existencia de procesos discriminatorios, presente en nuestras hipótesis iniciales, se acababa de confirmar cuando ganó dimensiones mayores a las habíamos previsto. Aunque difícilmente aparecían en el inicio de las entrevistas, escenas y relatos de discriminación y prejuicio, en diferentes grados y dinámicas, marcaron todos los relatos de madres y estudiantes, profesores y personas dentro y fuera del colegio. Así el tono general de la entrevista intentara no resaltar la existencia de conflictos, situación ejemplificada en frases como "todo está bien, vivimos y estamos bien integrados aquí en São Paulo y en el colegio", como fue el caso de E5, que pareció estar satisfecha con la trayectoria escolar y con las perspectivas futura, el tema aparecía y, en la gran mayoría de veces, estaba junto a relatos de experiencias personales. En el caso de esta estudiante, esto apareció cuando ella narró la dinámica de los grupos brasileros y bolivianos:

Son grupitos, no, y de repente llegan los brasileros queriendo saber: "¿de qué están hablando?". Y nosotros decimos "nada". Pero ellos piensan que porque nosotros nos estamos riendo, nos estamos riendo de ellos, iviste? No tiene nada que ver. Entonces llegan, a veces pelean, molestan... Un día yo les dije que nosotros contribuíamos con su país.

El joven E4, de 18 años, que igualmente tenía un discurso marcado inicialmente por el "Todo está bien", sin detenerse en los conflictos generados por su presencia en el grupo de EJA de una escuela municipal, al final de la entrevista reveló que normalmente sufre de discriminación: "Yo tengo un poquito de miedo de equivocarme con el portugués y ser discriminado por eso, sí, porque hay personas allá en Brasil que dicen "Ey extranjero, qué estás haciendo aquí, deberías ser deportado para Bolivia".

Desde la perspectiva de los profesores, esta dinámica es percibida y relatada como una discriminación que se manifiesta claramente en los salones de clase. Como relata la profesora P1 o el director D1: "En el salón de clase los niños decían: "ah, ese boliviano de ahí, profesor". Yo decía, "no, él es Cristian o Vilmar, etc.", para poder ir quitando eso (D1). 
Los argumentos son los recurrentes. Que ellos no deberían estar aquí, cuando ellos consiguen dinero para ir para otros lugares, o regresar a Bolivia o ir a Argentina, dicen: "Uy qué bueno que ellos se fueran [...]". Por ejemplo, estoy explicando otra cosa que no tiene nada que ver y ellos dicen: "Ey, quítate bolivia, porque deberías regresar para tu tierra en vez de estar ahí molestándome". $Y$ entonces no se ve reacción $(\mathrm{P} 1)$.

\section{Otro profesor es bastante explícito:}

Ellos deben reproducir lo que los padres hablan en casa. Parece que la relación en el barrio es tensa, la relación del espacio, de cómo están tomando nuestros empleos. [...] Dicen: "ah, profesor, ellos ensucian todo, ipor qué no regresan al país de ellos? Dicen que los bolivianos no se bañan, está ese problema. Y se mantienen alejados. [...] A veces me siento desesperado también, no voy a decir que sé cómo trabajar con todas esas cosas porque no es verdad, pues estamos hablando de una cosa nueva. Es diferente, por ejemplo, de la cuestión del negro, pues yo soy negro, tengo una bibliografía, ya leí mucho hasta para conocerme. Pero en relación a la América Española, soy totalmente ignorante (P3).

La joven E6, que tiene 19 años y no va al colegio en Brasil, contó el caso de su hermano, de 7 años. Después de sufrir situaciones de violencia y presenciar otras en el colegio donde estaba, la madre decidió mandarlo otra vez para Bolivia. El caso más extremo que nos fue relatado, fue el de un joven boliviano que fue agredido hasta morir dentro del colegio:

E6 -Es que ahí había mucha discriminación. El niño traía cositas así dulces, y los brasileros se las quitaban, entonces un día él no se las quiso dar. Creo que él estaba enfermo, que tenía alguna cosa en el estómago, un tumor, no sé. Y ese día ellos le pegaron hasta acabarlo.

M3 -no fue una vez, le pegaron varias veces. Solo que el niño le contaba a la mamá y la mamá tímida no dijo nada, dejó al niño, no le dio importancia. [...] Él (el hijo) quedó muy traumado, tenía 7 años y también se calló. Entonces cuando se murió el niño, creo que hicieron reunión con los padres, pero como la madre era boliviana, ella no hizo nada, no dijo nada. Yo no fui hasta allá, fue mi hijo el que me contó todo [...]. Muchos niños bolivianos no querían ir más al colegio, mi hijo me decía "me van a pegar a mí y voy a morir". Yo le decía que no, que no iba a morir, que tenía que saber defenderse. Yo fui con él, entonces vino no sé si era directora o algunas profesoras y les dije "mi hijo no quiere venir a la escuela, ¿qué pasa?". Ella dijo: "aquí no pasó nada".

E6: Creo que fue un señor allá en la escuela, no sé quién era, creo que del Ministerio de Educación, a decir que aquello no podía ser, tanta discriminación, había muchos padres que estaban así en contra. Algunos decían que los bolivianos tenían que tener una escuela aparte, pero ese señor quería unirnos, que todos somos iguales, decía que tenía que ser la misma escuela, yo sé porque fui a esa reunión después de que murió el niño. Había mucha discriminación antes. Ahora ya no hay tanta. 


\section{La escuela brasilera en la visión boliviana}

Nuestro tercer eje de análisis es sobre las familias y sus expectativas en relación al colegio. ¿Qué espera la familia inmigrante boliviana del colegio? ¿Qué dicen las madres sobre la relación con el colegio, con los profesores, sobre la permanencia, el éxito o el fracaso escolar?

Con base en esto, iniciamos una escucha atenta, en especial de las madres con las que conversamos, y una primera característica que surge es una indignación, por parte de ellas, sobre la dinámica de las escuelas brasileras. Aquí, los alumnos no respetan el profesor, las madres no los saben educar para eso, no llevan los estudios en serio:

El problema son los niños brasileros que no tienen educación, no respetan a la profesora, no hacen la tarea. Cuando la profesora toma la lección, ellos dicen "no la hice". ¿Cómo así? Mi hija que ya había estudiado en Bolivia, queda extrañada porque en Bolivia el estudio es estricto. ¡No le puede contestar a una profesora así! Aquí no, mi hija estaba muy sorprendida, aquí no respetan. Entonces la profesora, como no puede ser más paciente, los regañaba y al día siguiente estaba ahí la madre diciendo "¿por qué le gritó a mi hija?". A veces creo que los papás también están equivocados (M3).

La característica del respeto y de una exigencia mayor en relación al desempeño escolar, aparece de otra manera en el discurso de los profesores. En general, ven a las madres bolivianas como presentes y participativas, y sospechan que vinieron de una cultura en la que el irrespeto al profesor o a la institución escolar es inaceptable:

Están bastante presentes. [...] El año pasado le di un NS (No Satisfactorio) a un niño y ella vino a hablar, quería saber por qué había pasado eso. [...] Exigen autoridad, una madre boliviana una vez me contó que de donde ella venía el profesor hasta podía pegarle al alumno (P2).

Los padres bolivianos no aceptan notas bajas. Noto también, que esa actitud revela un carácter de la propia cultura local. Parece que se tiene cierta reverencia con el profesor que no hay aquí. Y los hijos reproducen (P3).

Un elemento que apareció en el discurso de una de las madres entrevistadas, es el sueño de que existiera una escuela separada. Por la descripción de ella, se refería a una escuela con el modelo de las instituciones privadas que se esfuerzan por el atendimiento específico a algunas nacionalidades:

Sabe, a veces yo quisiera una escuela aparte, sólo para los bolivianos, pero usted sabe, boliviano no es unido. En La Paz, hay escuela de brasileros, de alemanes, de americanos. Entonces a veces pienso que sería bueno una escuela para bolivianos, así no habría problemas para mi hijo, porque el idioma más que todo... [...] Por ejemplo, si estoy aquí por tres años, cinco años, hay mucha gente que viene a 
trabajar aquí y viene con los hijos, y los hijos tienen que ir a la escuela, y claro aquí escuela hay, pero existe ese problema del idioma, entonces cuando uno regresa allá a Bolivia ellos no quieren ni escribir, uno tiene que presentar muchas cosas.

En cuanto a las expectativas con relación al estudio, M2, madre de cuatro hijos dijo: "él va a la escuelita de adultos en la noche, niña, para poder salir más rápido del colegio, para poder estudiar otra carrera, él quiere ser alguna cosa, abogado, médico, como todo joven aspira a ser lo mejor en la vida". La manera como los hijos respondieron fue otra:

E3 -Yo ya hice un curso de programador de computador. Ahorita quería hacer la universidad, pero ellos no validan. Ellos piensan que me voy, que no voy a vivir más aquí.

E2 -Yo quería trabajar con carros, hacer un curso de automotor.

E4 -Yo de informática también.

En el caso de E6, de 19 años, que estaba fuera de la escuela, la idea inicial era seguir en el ramo de la costura: "Pensé en trabajar con algún coreano, pensé en hacer costura, ser práctica", afirmación que desagradó a la madre: "Yo le dije que hiciera otra cosa, pero no se anima a buscar". En ese momento, la joven repitió lo que la madre venía diciendo hasta entonces, el discurso sobre el miedo de salir a la calle, miedo del colegio, miedo de un trabajo nuevo:

Es que tengo un poquito de miedo, que el jefe sea un brasilero o un coreano, son diferentes, tengo un poquito de miedo. Yo no era así, pero ahora tengo un poco. Tengo un amigo que fue a trabajar en una panadería y trabaja por dos, pero gana por uno. [...] pero va a ser bueno trabajar afuera, porque cansa, todos los días la misma cosa.

Finalmente, en el caso de E5, que está terminando la Educación Media, la perspectiva de ella era de hecho hacer una carrera en São Paulo, al contrario de muchos compañeros que hacen los cursos técnicos o ingresan directamente en el marcado de confección. Después, pensó bien y cambió de idea:

Mi sueño no es la USP, es la PUC. Tengo dos amigos que estudian allá y otros que estudian en la USP. Ellos fueron voluntarios de la Amnistía ahora y trabajaron en el CAMI, estudian relaciones internacionales. La verdad, yo también quería estudiar relaciones internacionales.

\section{Algunas conclusiones}

En este estudio, buscamos entender cómo está siendo realizado el derecho humano a la educación para los y las inmigrantes de Bolivia que viven en São Paulo, y un primer paso es, ciertamente, quitar el tema de la invisibilidad. Poco sabemos sobre 
los bolivianos(as) que están en los colegios. Son poco notados, poco percibidos, desconocidos. En consecuencia, en las políticas públicas nos hay acciones enfocadas que consideren sus especificidades en ninguna esfera (municipal, estadual o federal), nivel o modalidad de educación.

La universalización del derecho a la educación es atravesada por diversas tensiones. Es un hecho que la educación está disponible, en el sentido en que hay escuelas y está la legislación que permite que la matrícula sea efectuada, sea cual sea el estatus de la persona en el País. Sin embargo, la distancia entre lo que está garantizado legalmente y lo que se manifiesta en lo cotidiano es un desafío en todos los campos de los Derechos Humanos, y en nuestro estudio, esto no fue diferente.

El acceso a la educación es un primer factor que pone en jaque su principio de universalidad, en la medida en que hay barreras para los inmigrantes. Para aquellos en situación irregular en el país, aunque la ley garantice ese derecho, la falta de documentos es un obstáculo para entrar en cursos o colegios, para cambiar de colegio (cuando no se facilita el historial académico) e incluso para salir (sin la emisión del certificado de conclusión del curso). Especialmente en el caso de los que no tienen documentos, la burocracia y la falta de información sobre los derechos educativos son las barreras que parecen más evidentes.

Superando esa primera frontera y una vez dentro de la escuela, la mirada sobra aceptabilidad de esta educación brindó elementos importantes que caracterizan un ambiente que promueve poco el aprendizaje y desafía la promoción de los derechos humanos. De un lado, niños y niñas sin nombre, Ilamados "Bolivia" por los compañeros y cuya voz rara vez es escuchada por los profesores, fueron descritos como callados, retraídos, silenciosos, hablan poco, participan poco. ¿Y qué comunica este silencio? ¿Miedo? ¿Indiferencia? ¿Respeto a la educación escolar y reverencia al/a la profesor/a? Por otro lado, los compañeros, profesores, empleados del colegio, que saben muy poco de ellos y ellas. En un primer momento, esa presencia parece despertar una relación ambigua: parecen estar cerca y lejos al mismo tiempo, pero aún son extraños, en cualquier caso. Y, si causan extrañamiento, dice Mafesolli (2001), es porque ya hacen parte del grupo. Esto, como ya decía Sayad (1998), ocurre cuando los brasileros comienzan a verlos(as) ahí, al lado, reivindicando cualquier cosa, además de servir como mano de obra en un sector que los brasileros(as) no estarían dispuestos a ocupar. De a pocos, compartirán el mismo espacio, en nuestro caso el mismo colegio, la misma calle, el mismo punto de salud. En estos (des) encuentros escuchamos discursos como "están robando nuestros trabajos", "¿Por qué no regresan a Bolivia?". Muchos reproducen dentro del colegio la relación conflictiva que establecieron en el barrio: insultos, provocaciones, episodios de violencia. La discriminación relatada en las entrevistas evidencia una relación tensa, con los profesores sin saber qué hacer, con pocos elementos para intervenir. 
En lo que respecta a la adaptabilidad de ese derecho, ¿cómo es posible considerar las especificidades de alumnos y alumnos que ni siquiera son notados? En este punto, el idioma es un problema inicial para ser pensado, pues se configura como un impedimento: primero es necesario "acostumbrarse con el portugués" y solo después buscar el colegio. ¿No sería razonable pensar en clases de refuerzo, visto que estos y estas estudiantes no están en igualdad de condiciones con los demás? Por otro lado, existe la cuestión del idioma nativo, ¿qué lugar ocupará? ¿Tiene que ser olvidado? Ese "olvido", en el relato de las entrevistas, es un rasgo que los identifica como bolivianos(as), y, ante el escenario de discriminación, es mejor esconderlo.

Uno de los temas contemporáneos de las grandes ciudades es cómo compartir el espacio. Los inmigrantes, en este contexto de desplazamiento entre países, comienzan a vivir ahí al lado, como dice Bauman (2008, p. 35), sin "haber sido invitados". Al observar esa relación en diferentes lugares del mundo, el autor constató que "los extranjeros tienden a parecer más atemorizantes cuanto más distantes, desconocidos e incomprensibles los vemos". En nuestro estudio, a excepción de grupos bastante restrictos, percibimos un diálogo todavía pequeño, tímido e insuficiente. La convivencia en los espacios públicos es limitada, las fronteras son reales. Romper esa lógica, interviniendo la dinámica y provocando el reconocimiento, es uno de los desafíos que encontramos. Pero, ¿cuál es la posibilidad de una experiencia compartida, si no se comparte el espacio de vida? ¿Una experiencia que vaya más allá de una convivencia ahí, lado a lado, pero en una combinación real de las diferencias en una unidad de determinada vida colectiva?, como indaga Touraine (1998). El colegio público es uno de los pocos espacios compartidos, es allí donde algunas barreras deberían ser derribadas.

Frente a la experiencia violenta que su hijo vivió dentro de un colegio y también por trabas causadas por el idioma, una de las madres propuso "la escuela separada". Sería una escuela solo para bolivianos y bolivianas, revelando, así, los dilemas alrededor de la aceptabilidad (calidad) de la escuela. La idea, contó ella, habría nacido en una conversación con otras madres, tanto bolivianas como brasileras, estas porque creían que eran los inmigrantes los que estaban molestando. ¿Sería, así, una propuesta definitiva para la resolución de los conflictos? A nuestro modo de ver y desde una perspectiva de los Derechos Humanos, la idea de segregación debería ser rechazada. Recordamos que el gran desafío contemporáneo no es aceptar los alejamientos y las segregaciones, por edad, generaciones, sexo o sexualidad, raza, etnia, religión clase o grupo de status social o profesional. Más que nunca, es importante criticar los guetos, los enclaves fortificados, el "no hables con extraños" (Schilling, 2008).

Para finalizar, volvamos al principio, cuando situamos esta investigación como parte de los debates sobre los desafíos de la universalización de los derechos en sociedades desiguales y discriminatorias. La búsqueda de la realización plena del 
derecho humano a la educación incluye, como dijimos, además del acceso en sí, la preocupación por el modo como se da la permanencia en el sistema. Su realización se lleva a acabo si las dimensiones de disponibilidad, accesibilidad, aceptabilidad y adaptabilidad están protegidas, realizadas y promovidas de manera conjunta.

El camino para la realización completa del derecho a la educación para los y las inmigrantes de Bolivia, atraviesa todos esos desafíos y, a nuestro modo de ver, puede comenzar con un proceso de escucha y amplificación de sus voces. 


\section{Referencias}

Bauman, Z. (2005). Confianza y temor en la ciudad; vivir con extranjeros. Barcelona: Arcadia.

Bonassi, M. (2000). Canta, américa sem fronteiras! Imigrantes latino-americanos no brasil. São Paulo: Loyola.

Ley federal n. 8069/90.(1990, 13 de julio). Brasil.

Ley 6815. Ley del extranjero. Artículo 48. Brasil.

Constitución federal. (1988). Brasil.

Convención internacional sobre la protección de todos los trabajadores migrantes y sus familiares. Artigo 30.

Cymbalista, R., Rolnik, I. (2007). Comunidade boliviana em são paulo: definindo padrões de territorialidade. Cadernos Metrópole. (17), p. 119-133.

Consejo estadual de educación. Deliberación 16/1997. Brasil.

Consejo estadual de educación. №633/2008. Estado.

Resolución 9. (1994). Estado de Brasil: Secretaría de educación del estado de são paulo.

Resolución. (1995). Estado de Brasil. Secretaría de educación del estado de são paulo.

Fernandes, F. (1961). Folclore e mudança social na cidade de são paulo. São paulo: Anhembi.

lanni, O. (2004) Capitalismo, violência e terrorismo. Rio de Janeiro: Civilização Brasileira.

International Organization for Migration (IOM). (2008). World migration report 2008: managing labour mobility in the evolving global economy. Genebra: IOM.

Lafer, C. (1988). A reconstrução dos direitos humanos: um diálogo com o pensamento de hannah arendt. São Paulo: Companhia das Letras.

Alto comisionado de las naciones unidas para los derechos humanos. (2006). Los derechos de los no ciudadanos. Nueva York: Ginebra.

Maffesoli, M. (2001). Sobre o nomadismo - vagabundagens pós-modernas. Rio de janeiro São Paulo: Record.

Martins, J. (2001, nov.). Por uma pedagogia dos inocentes. Tempo social, rev. Sociologia usp. 13(2), p. 21-30..

Dictamen 17. (2004). Município, Consejo municipal de educación.

Muñoz, V. (2010). The right to education of migrants, refugees and asylum-seekers. Reporte presentado al consejo de derechos humanos de la ONU. Ginebra, New York: Un General Assembly.

Observación general n 13 sobre el derecho a la educación. (1999). Comité de derechos económicos, sociales e culturales (desc) de las naciones unidas.

Oficina del alto comisionado de las naciones unidas para los derechos humanos. (2006). Los derechos de los no ciudadanos. Nueva york. Ginebra: Naciones Unidas.

Câmara Municipal de São Paulo. (2006). Relatoría final de la comisión parlamentar de investigación para eliminar la explotación del trabajo similar al esclavo en la ciudad de São Paulo. Câmara Municipal de São Paulo.

Santos Júnior, B. (1980). Os direitos humanos e alguns aspectos da situação do estrangeiro. Revista do advogado. (1) 3, p. 50-53. 
São Paulo (municipio). (2006). Relatoría final de la comisión parlamentar de investigación para eliminar la explotación del trabajo similar al esclavo. São Paulo: câmara municipal.

Sayad, A. (1998). A imigração. São Paulo: Edusp.

Schilling, F. (2008). O direito à educação, um longo caminho. En: Bittar, E. C. B. (Ed.). Educação e metodologia para os direitos humanos. São paulo: quartier latin.p. 273-283.

Silva, S. (2006, mayo-agosto). A. Da. Bolivianos em são paulo: entre o sonho e a realidade. Dossiê migrações. 20(57), p. 157-170.

Silva, S. A. Da. (2005), Os imigrantes e os direitos humanos no brasil. En: Serviço pastoral do migrante (Ed). Travessia da desordem global - fórum social das migrações. São Paulo: Paulinas.

Silva, S. A. Da. (2005). Bolivianos: a presença da cultura andina. São Paulo: companhia editora nacional.

Silva, S. A. Da. (1997). Costurando sonhos: trajetória de um grupo de imigrantes bolivianos em São Paulo. São Paulo: Paulinas.

Silva, S. A. Da. (2003). Virgem, mãe, terra. Festas e tradições bolivianas na metrópole. São Paulo: Hucitec - Fapesp.

Sousa Santos, B. (2006). A gramática do tempo - para uma nova cultura política. São Paulo: Cortez.

Tomasevski, K. (2006). Human rights obligations in education: the 4-a scheme. Nijmegen, The Netherlands : Wolf Legal Publishers.

Touraine, A. (1998). Poderemos viver juntos? Iguais e diferentes. Petrópolis: Vozes. 